\title{
Meperidine-induced hypereosinophilia: Report of a case
}

\author{
M.B. CLEARFIELD, D.0
}

Fort Worth, Texas

\begin{abstract}
Drug-induced eosinophilia
has been reported frequently in the literature. The following case study suggests a hypereosinophilic reaction secondary to meperidine use, which resolved without adverse sequelae after discontinuation of the medication. Hypereosinophilia is uncommon and has not been reported in association with meperidine.
\end{abstract}

Elevations in the total eosinophil count have been frequently reported in the literature. However, a marked hypereosinophilia associated with meperidine use has not been previously documented. The following case details this adverse response to a commonly prescribed medication.

\section{Report of case}

A 42-year-old Caucasian man was evaluated on November 15, 1982, for pain and paresthesias of his left arm after a fall. Radiographic evaluation revealed no evidence of fracture, and initial therapy included local manipulation and analgesics (acetaminophen with codeine). However, 2 weeks after the precipitating event, the pain and paresthesias had increased to the point where hospitalization was required.

The prior surgical history consisted of repair of the right medial meniscus of the knee in 1958 and minor eye surgery in 1976. There was no history of hematologic abnormalities or use of parenteral medications during prior hospitalizations. The patient's past medical history included peptic ulcer disease in 1962 and a prostatic infection 7 months prior to admission. The systems review was negative. The social history was positive for 50 pack years of cigarettes and occasional alcohol consumption. The patient denied atopic disease, allergy, and dermatosis, and he had no pets. His travel history was negative for the past 5 years; prior to that he had lived in Venezuela for 2 years and had also traveled to Southeast Asia and Mexico. The patient worked as an oil rigger and denied environmental exposure.

Physical examination revealed a well-nourished man with normal vital signs. Abnormalities included global hypalgesia and decreased strength of the left arm compared to the right, without associated atrophy, fasicula- tion, or tremor. A somatic dysfunction was noted in the cervical paravertebral area on the left. The initial leukocyte count was $8,600 / \mathrm{cu}$. mm., with 3 percent eosinophils. Laboratory values for hemoglobin, hematocrit, prothrombin time, partial prothrombin time, rapid plasma reagin, electrolytes, Westergren sedimentation rate, calcium, phosphate, glucose, creatinine, albumin, total protein, bilirubin, and alkaline phosphatase all were normal, as were urinalysis results.

A computed tomography (CT) scan without contrast medium revealed large posterior osteophytes of the cervical spine. Myelography with iophendylate (Pantopaque) confirmed nerve root compression at the C5-6 and C6-7 levels. Following myelography, there was adequate retrieval of the contrast media from the subarachnoid space, with only a small amount persisting at the level of the first sacral vertebra.

Initial medications included diazepam (Valium, 10 mg. 4 times daily) and oxycodone hydrochloride with aspirin (Percodan, $4.5 \mathrm{mg}$. every 3 to 4 hours). Meperidine (Demerol) in varying doses with and without hydroxyzine pamoate (Vistaril) was prescribed for additional pain relief, and temazepam (Restoril) was ordered for sleep. Naproxen (Naprosyn, $375 \mathrm{mg}$.) was given twice daily.

A second complete blood count on December 2 showed a leukocyte count of $13,000 / \mathrm{cu}$. mm., with 18 percent eosinophils; on December 6, the leukocyte count was $26,500 / \mathrm{cu}$. mm., with 38 percent eosinophils, and there was no change in the hemoglobin concentration, hematocrit reading, or platelet count. Although the patient was still in significant pain, physical examination findings remained unchanged. On December 7 , multilevel cervical microneurosurgical laminectomy was performed without complication. On December 9, a repeat complete blood count again showed an elevated leukocyte level of $25,800 / \mathrm{cu}$. mm., with 48 percent eosinophils, a quantitated eosinophil count of 12,267 , and Westergren sedimentation rate of $45 \mathrm{~mm}$. $/ \mathrm{hr}$. Temazepam, naproxen, and oxycodone hydrochloride were discontinued. The physical examination findings did not change, except for a transient fever of 38.4 C. (101.2 F.), which resolved without specific therapy in 48 hours. Re-evaluation revealed no evidence of muscle pain, swelling, rash, petechiae, or cardiac or pulmonary abnormalities. The chest $\mathrm{x}$-ray and electrocardiogram remained within normal limits. The patient slowly improved clinically, although the leukocyte and eosinophil counts continued to rise. On December 16, the leukocyte count was $29,100 / \mathrm{cu}$. 
mm., with 66 percent eosinophils, and the meperidine and hydroxyzine pamoate were discontinued. Further testing included negative results for rheumatoid factor, $\mathrm{C} 1 \mathrm{Q}$ and raji cell immune complex assay, cryoglobulin, blood ethanol, febrile agglutinins, $\mathrm{HbsAg}$, antifilarial antibody by complement fixation for both trichinosis and Echinococcus, and stools for ova and parasites. Other normal studies were those for serum protein and immunoprotein electrophoresis, prothrombin time, partial thromboplastin time, fibrinogen level, bleeding time (Ivy's method), acid phosphatase, leukocyte alkaline phosphatase, serum B12, serum IgE, and intermediate purified protein derivative of tuberculin.

Lumbar puncture was performed on December 17, with the following results: protein, $70 \mathrm{mg}$./dl.; glucose, $54 \mathrm{mg}$./ dl.; 1 lymphocyte and no eosinophils; and Gram's stain, no organisms. Cultures of blood, urine, and cerebrospinal fluid showed no growth. Bone marrow aspiration and biopsy revealed a hypercellular bone marrow of chiefly myelocytic and metamyelocytic eosinophilic forms. Erythropoiesis appeared to be normoblastic, and the remaining myeloid forms were quantitively normal. A number of eosinophilic precursors showed significant cytoplasmic vacuolization, with no evidence of maturation arrest or neoplastic infiltrate. Prussian blue stain demonstrated adequate iron stores, and chromosome analysis was normal.

.The patient had slow neurologic improvement of the involved arm. Because of persistent pain, oxycodone was reinstituted (December 17-24); it was then replaced by propoxyphene hydrochloride (Darvon, $65 \mathrm{mg}$.). Slight decreases in the leukocyte count $(23,800 / \mathrm{cu}$. mm.) and eosinophilia (61 percent) were noted on December 21 , and this trend continued until discharge. On December 28, skin tests were performed utilizing $1 \mathrm{cc}$. of xylocaine, $1 \mathrm{cc}$. of iophendylate (Pantopaque), and $1 \mathrm{cc}$. of meperidine subcutaneously. Within 24 hours, a $12-\mathrm{mm}$., indurated wheal was noted only at the meperidine injection site.

The patient was discharged on December 30 with the leukocyte count normalized $(7,100 / \mathrm{cu} . \mathrm{mm}$.) and the eosinophil count decreasing ( 20 percent). At hospitalization 5 months later for an unrelated problem, the leukocyte and differential counts were normal. During this second hospitalization, the patient was medicated with hydroxyzine pamoate, piroxicam (Feldene), and diazepam without subsequent eosinophilia. The complete blood count was repeated 18 months after the initial hospitalization, with leukocyte and eosinophil counts being normal. There was no evidence of any organ damage from the previous hypereosinophilia.

\section{Discussion}

The differential diagnosis of eosinophilia has intrigued diagnosticians for a century. Most reviews of eosinophilia include minimal eosinophil elevations; however, an updated version of the diseases covered in the 1944 Mayo Clinic review ${ }^{1}$ of 418 patients with 20 percent or more eosinophils is presented in Table 1 . In the case under discussion, there is no history of atopic disease or chronic epidermal disease. The negative examination of the
TABLE 1. DISEASE WITH EOSINOPHILIA $>20$ PERCENT (UPDATED FROM STICKNEY AND HECK ${ }^{1}$ ).

Atopic disease

Lymphoproliferative disease

Dermatoses

Nonparasitic infections (principally streptococcal)

Periarteritis nodosa

Parasitic infections

Nonlymphoma malignant tumors and leukemia

Pulmonary eosinophilic syndromes

Idiopathic hypereosinophilic syndrome (HES)

abdomen, absence of lymphadenopathy, and negative bone marrow examination probably exclude lymphoma and leukemia. There was no history of exposure to parasites, and parasitic evaluation was negative. All cultures were negative and the patient remained afebrile, which would make an infectious etiology unlikely. The total absence of symptoms and radiographic evidence of pulmonary disease excludes eosinophilic pulmonary syndromes, and there was no clinical or laboratory evidence of connective tissue disorders. Further, the short duration of the eosinophilia and lack of cardiovascular, pulmonary, or dermal involvement excludes the idiopathic hypereosinophilia syndrome. ${ }^{3}$ The rapid evolution of the eosinophilia suggests an external etiologic factor.

Eosinophilia is a well-known side effect of drug treatment. ${ }^{3}$ In review of therapeutic agents as a cause of eosinophilia, including a text ${ }^{4}$ on the subject, none of the medications used in the treatment of this patient was noted as a cause of eosinophilia. There are, however, case reports of peripheral eosinophilia occurring after use of iodinated contrast media (irrespective of dosage or type); this typically peaked within 48 hours and persisted for approximately 6 days. ${ }^{5}$ When myelography has been implicated, it usually was manifested as cerebrospinal fluid eosinophilia. ${ }^{6}$ This patient did not have any cerebrospinal fluid eosinophilia, nor did he react to an intradermal injection of iophendylate, thus suggesting that this agent was not the cause of the eosinophilia. In most patients, hypersensitivity reactions from drugs occur after 2 weeks of treatment, which suggests an immune response. ${ }^{3}$

Two main types of hypersensitivity underlie most drug-associated eosinophilic reactions. The less common IgE-mediated (type I) reaction seldom causes chronic tissue injury and is an important cause of anaphylactic reactions. ${ }^{3}$ The normal $\operatorname{IgE}$ level and slowly progressive eosinophilia suggest that this was not the likely reaction in this case. ${ }^{7,8}$ The T-lymphocyte-dependent (type IV) reactions can lead to chronic and, occasionally, to irreversible 
tissue damage. ${ }^{4}$ It has been proposed that tissue damage secondary to eosinophilia is associated with abnormal eosinophils. This patient was noted to have vacuolization of the bone marrow eosinophils (fulfilling the criteria of 10 or more distinct vacuoles in the cytoplasm of 50 percent of the eosinophils. $)^{1-9}$ However, the criteria for vacuolization or hypogranular eosinophils was not met in the peripheral blood smear.

The significance of this finding is questionable, because there was no evidence of tissue damage acutely or at later hospitalization. However, the more common cell-mediated hypersensitivity reaction does seem to be implicated. The positive skin test for meperidine suggests a delayed type of sensitivity to this medication. ${ }^{10-11}$ Temazepam and naproxen were administered prior to onset of the eosinophilia and discontinued before resolution after low cumulative doses. A second group of drugs (diazepam, oxycodene hydrochloride, hydroxyzine pamoate) were reintroduced at a later date or subsequent hospitalization without recurrence of eosinophilia; therefore, these drugs are unlikely causative agents. The only remaining medication is meperidine. Other data implicating meperidine include the positive skin test, the high cumulative dosage of the medication coinciding with the progressive eosinophilia, and the resolution of the eosinophilia after discontinuation. Although the patient was not rechallenged with meperidine, evaluation at 6 and 18 months revealed no evidence of leukocytosis or eosinophilia.

\section{Comment}

The clinical presentation in this case suggests a cell-mediated hypersensitivity reaction to meperidine. Hypereosinophilia has not been pre- viously reported as a side effect of this medication. Both the Food and Drug Administration and the company that manufactures meperidine (Winthrop-Breon) had no record of meperidine-related hypereosinophilia. Because this medication is frequently prescribed for analgesia in a hospital setting, further evaluation is necessary to confirm meperidine as a cause of idiopathic eosinophilia.

1. Stickney, J.M., and Heck, F.J.: The clinical occurrence of eosinophilia Med Clin North Am 28:915-9, Jul 44

2. Fauci, A.S., et al.: NIH conference. The idiopathic hypereosinophilic syndrome. Clinical, pathophysiologic, and therapeutic consideration Ann Intern Med 97:78-92, Jul 82

3. Spry, C.J.: Eosinophilia in allergic reactions to drugs. Clin Haematol 9:521-34, Oct 80

4. Swanson, M. and Cook, R.: Drugs, chemicals and blood dyscrasias Drug Intelligence Publication, Bethesda, Maryland, 1977, pp. 1124-5

5. Plausic, B., et al:: Eosinophilia caused by iodinated radiographic contrast media. Clin Radiol 639-42, 1983

6. Holley, H.P., and Al-Ibrahim, M.S.: CSF eosinophilia following myelography. JAMA 242:2432-3, 30 Nov 79

7. Somorin, A.O., Heiner, D., and Hunponu-Wusu, D.O.: Correlation of blood eosinophils and total serum IgE. Cent Afr J Med 24:255-7, Dec 78 8. Halomen, M., et al: An epidemiologic study of the interrelationship of total serum immunoglobulin E, allergy skin test reactivity, and eosinophilia. J Allergy Clin Immunol 69:221-8, Feb 82

9. Schatz, M., Wasserman, S., and Patterson, R.: The eosinophil and the lung. Arch Intern Med 142:1515-9, Aug 82

10. Pmia, L., et al.: Heterogeneity of human blood eosinophils. Relation to the level and origin of hypereosinophilia. Int Arch Allergy Appl Immunol 72:336-46, 1983

11. Barbee, R.A., et al.: Immediate skin-test reactivity in a general population sample. Ann Intern Med 84:129-3, Feb 76

Barbee, R.A., et al.: Distribution of $\mathrm{IgE}$ in a community population sample. Correlations with age, sex, and allergen skin test reactivity. $J$ Allergy Clin Immunol 68:106-11, Aug 81

Dr. Clearfield is chairman and associate professor of medicine, Department of Medicine, Texas College of Osteopathic Medicine, Fort Worth.

Dr. Clearfield, TCOM, Camp Bowie at Montgomery, Fort Worth, Texas 76107 . 
